\title{
Textilloma Mimicking an Intracranial Postoperative Abscess
}

\author{
Giovanni Tringali ${ }^{{ }^{*}}$, Morgan Broggi ${ }^{1}$, Giuseppe Didato ${ }^{2}$, Ludovico D'Incerti ${ }^{1}$, Angelo Franzini ${ }^{1}$ and Flavio Villani $^{2}$ \\ ${ }^{1}$ Department of Neuroradiology, Fondazione Istituto Neurologico Carlo Besta, Milan, Italy \\ ${ }^{2}$ Department of Neurology and Neuropathology, Fondazione IRCCS Istituto Neurologico Carlo Besta, Milan, Italy \\ *Corresponding author: Giovanni Tringali, Department of Neurosurgery, Fondazione IRCCS Carlo Besta, Milan, Italy, Tel: +39223942421; Fax: +39270638217; E-mail: \\ giovannitringali@libero.it
}

Rec date: Jan 07, 2015, Acc date: Feb 18, 2015, Pub date: Feb 20, 2015

Copyright: $\odot 2015$ Tringali G, et al. This is an open-access article distributed under the terms of the Creative Commons Attribution License, which permits unrestricted use, distribution, and reproduction in any medium, provided the original author and source are credited.

\begin{abstract}
Background: Achieving haemostasis during surgical procedures within the brain is of paramount importance. Bipolar cauterization is most effectively used to occlude identifiable vessels but it has minimal efficacy in controlling the oozing bleeding that characterises most neurological pathologies. In such cases chemical haemostatic agents are often preferable to bipolar cautery. Rarely these chemical agents may produce a foreign-body reaction that resembles infectious or malignant processes. On imaging significant oedema and ring-like enhancement on postcontrast studies may falsely confirm the suspect of such processes, requiring new surgical intervention.
\end{abstract}

Methods: In this report a reaction to a chemical haemostatic agent in a patient operated on for a low grade glioma causing focal epilepsy is discussed.

Results: After a two year follow-up the patient is seizure free without neurological deficits, and MRI scans do not show any significant focal alteration except for surgical scar.

Conclusions: The literature reports sporadic cases of foreign-body reaction to haemostatic agents that may mimic neoplastic recurrency or post-surgical infectious complications on imaging studies, but this complication is still considered exceedingly rare. Our case, however, confirms this possibility, raising the suspicion that it may be overlooked when imaging studies are not performed soon after surgery or if the reaction happens in a silent cerebral area.

Keywords: Textilloma; Intracranial abscess; Foreign body reaction; Tumour recurrence; Chemical haemostats

\section{Introduction}

Whatever the surgical procedure is, one of the main concern for a surgeon is to achieve haemostasis. A clear operative field reduce the duration of the intervention and the risk of injuries to the surrounding structures. The most common method used to control bleeding is by bipolar cauterization, which is used to occlude identifiable vessels. When this is not sufficient or the bleeding is a continuous seepage in the surgical cavity, chemical haemostatic agents (CHA) may be used. Although, CHA are safe and widely used, rarely they may be responsible for an inflammatory reaction similar to those reported for a foreign body. The reaction described herein is a diagnostic challenge due to the difficult on MRI interpretation when it occur in gliomas.

\section{Case}

A 49-year-old male, suffering from left limbs focal motor seizures, was considered for neurosurgical treatment, after a complete presurgical evaluation (high field MRI, video EEG, PET, neuropsycological tests). His MRI feature was (Figure 1): right frontal precentral lesion round shape, sharp margins, inhomogeneous hyperintensity on T2-w.i. very few perifocal oedema. No enhancement after contrast medium administration was observed (Figure 1). After an informed consent was obtained, a Keyhole craniotomy for the excision of the lesion, under general anaesthesia and intraoperative neurophysiological monitoring, was done. The histopathological findings were consistent with low grade glioma. Immediate post operative CT showed no surgical complications. The patient was discharged in good clinical conditions. A week later he presented a sudden onset of progressive left hemiparesis and was readmitted at our Institution. On MRI the surgical cavity was (Figure 2) hyperintense on T2 w.i, intense and homogeneous enhancement on T1 post contrast images associated with increased perifocal edema. DWI did not show reduction of diffusivity. Based upon the rapid neurological deterioration a reopening of his craniotomy was performed under general anaesthesia. The cortex exposed was reddish but no sign of abscess was found. Intraoperative video fluorangiography (ICG) with flow analysis (OPMI Pentero FLOW800, Carl Zeiss, Jena, Germany) revealed an alteration of the capillary permeability. The area corresponding to the pre-operative enhancing lesion was placed where the local haemostatic agents were left. It was completely removed and the craniotomy was closed in the usual manner. The patient had immediate full recovery with resolution of his neurological deficits. Upon gross inspection and microscopic inspection, the lesion was found to be an approximately $2.5 \mathrm{~cm}$ retained textilloma. Close MRI follow up demonstrated thinning of the surgical cavity, disappearance of enhancement on its margins and regression of oedema four months after re-operation (Figure 2). The neurological examination remained unremarkable. 


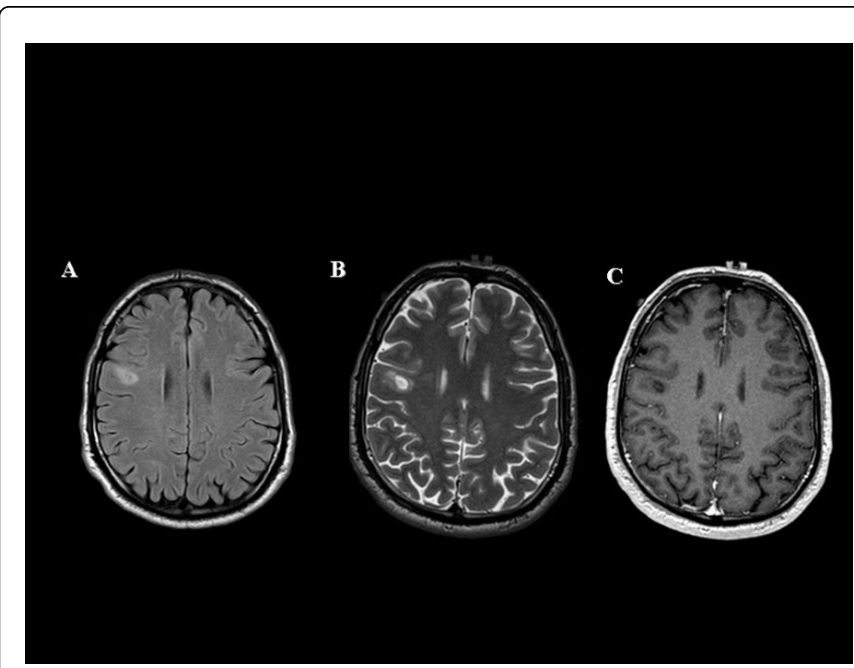

Figure 1: Pre-operative MRI study: Transversal FLAIR (A), T2 w.i. (B) and post contrast T1 w.i. (C) demonstrating hyperintense nonehancing small abnormality and little perifocal edema in the subcortical white matter of the right middle frontal convolution.
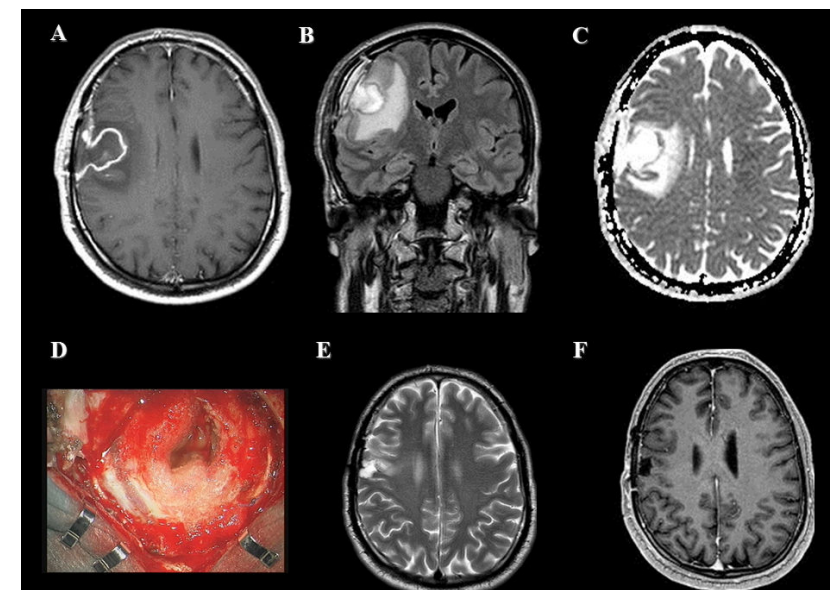

$\mathbf{E}$
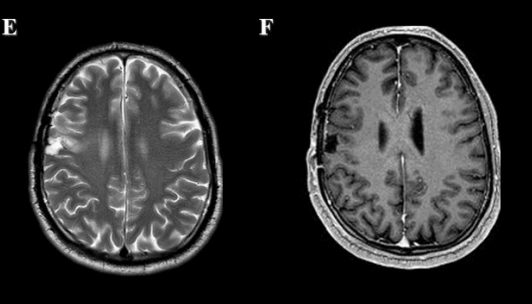

Figure 2: Post-operative MRI study: T1 w.i. post contrast (A), FLAIR coronal (B) and ADC (C) depiciting an area of enhancement along the border of the surgical cavity associated with perifocal edema and no reduction of diffusivity. Microscope magnification depicting the textilloma (C). Post surgical follow up after re-operation with regression of the perifocal edema (T2 w.i. E) and of the enhancement (on postcontrast T1 w.i. F).

\section{Discussion}

Bipolar cautery is widely used in any neurosurgical procedures because of its ability to selectively close small vessels. Nevertheless, bipolar cautery may cause dissipation of heat from the tips of the forceps inducing thermal injury to surrounding neural structures. Although the current output of electrosurgical generators can be modulated to deliver different waveforms to the tissue, spreading from the cut mode $(100 \%$ on) used to perform an enbloc resection through the more modulated ( $20 \%$ on) output to control heat dissipation, its use no matter how often applied, alter the blood-brain barrier. When the bleeding didn't occur from identifiable vessels, the wide use of bipolar cauterization could increase the risk of damage to the surrounding s structures. A less invasive tool is the use of CHA. The reason why they are left in situ is to decrease the risk of postoperative bleeding, which may occur as a consequence of blood pressure variations. Among these chemical agents oxidized regenerated cellulose (ORC) is one of the most commonly used. Following saturation with blood, it gradually increases in volume and forms a gelatinous mass that aids in the formation of the clot. Thus the mechanism of action is both related to the mass effect (gentle compression) and chemical. In the weeks later the deployment a reaction develop that lead to a complete adsorption of the ORC. Although CHA are considered safe, they are foreign substances introduced into the central nervous system. In rare cases a host reaction to these agents has been reported, mainly by general surgeon or in spinal procedures [1]. They may induce an excessive inflammatory reaction within the surgical site which can be silent or impair neurological function according to its site and volume. The conventional radiological features are challenging because the appearance is often indistinguishable from a recurrent tumour or an abscess. Almost all the cases reported in literature had an appearance as a ring like enhanced lesion associated with perilesional oedema. In such cases the most appropriate treatment is controversial, in fact little is known about the pathogenesis, and current treatment is mostly empirical guided by the neurological status. Our case had a progressive onset of motor weakness that guided our decision to revise the wound, but if we had the knowledge of this condition a more conservative approach would have been applied. A high dose steroids could have been proposed to avoid another surgery. Since the introduction of advanced MR imaging modalities, such as diffusion weighted imaging (DWI) with analysis of the apparent diffusion coefficient (ADC), several reports have been published dealing the differential characteristic of abscess, gliomas and recurrences [2]. Abscesses are reported to have a high signal on DWI and a reduced ADC within the cavity, while high grade glioma show a lower signal intensity on DWI and a higher ADC. In the case reported herein, the post surgical MRI resembled similar to a brain abscess, except for the absence of low ADC values. Although the absence of restricted diffusion should suggest for a recurrence, this hypothesis was not considered because of the hystopathological finding. Based on the above mentioned consideration conventional MRI is of little aid in the differential diagnostic process. When the MRI is observed in the early postoperative period it is affected by the vascular changes induced by the surgery itself [3]. The CT appearance of a CHA has been described [4] as mixed- or low-attenuation masses containing focal central collections of gas in earliest period, which [5] appear on conventional MRI (T2-weighted magnetic resonance with a short relaxation time) as a low signal intensity mass. Even the time lapse from the surgical intervention to the onset of the clinical sign do not address the correct diagnosis, it can vary from the immediate postoperative period to several years. In a case reported in literature [6] a [18F]fluorodeoxyglucose (FDG) positron emission tomography-computed tomography (PET/CT) disclosed uneven FDG uptake at the periphery of the mass. The authors based on the rim-shaped appearance, without significant central necrosis, compared to the global FDG uptake pattern of a tumour excluded the recurrences. Although the above mentioned paper refer to an abdominal textilloma when there's an high grade of suspicious a FDG-PET could improve the diagnostic process also in neurosurgery. 
Citation: Tringali G, Broggi M, Didato G, D'Incerti L, Franzini A, et al. (2015) Textilloma Mimicking an Intracranial Postoperative Abscess. J Neurol Disord 3: 213. doi:10.4172/2329-6895.1000213

Page 3 of 3

Re-absorbable haemostatic agents are used almost in all craniotomies at our institution and are usually left in situ. The amount of surgical procedures performed per year, at our institution, is about 3000 , thus the reaction described in this report represents a very rare complication occurred among a large number of patients (both spinal and cranial). It is opinion of the authors to continue to left these CHA in situ. The risk/benefit ratio remain in advantages compared to the risk of postoperative bleeding. What can be proposed, as consequence of this "side effect", is to report in the surgical report a description of the haemostatic agent that has been used, including its name, site, and amount. This information might be used as a reference in the interpretation of postoperative diagnostic images and help to identify the real incidence of inflammatory reaction due to $\mathrm{CHA}$.

\section{Disclosure}

The authors have no conflict of interest to declare

I, Giovanni Tringali, certify that this manuscript is a unique submission and is not being considered for publication with any other source in any medium.

All the Authors have taken part in the drawing of the manuscript.

\section{References}

1. Kothbauer KF, Jallo GI, Siffert J, Jimenez E, Allen JC, et al. (2001) Foreign body reaction to hemostatic materials mimicking recurrent brain tumor. Report of three cases. J Neurosurg 95: 503-506.

2. Desprechins B, Stadnik T, Koerts G, Shabana W, Breucq C, et al. (1999) Use of diffusion-weighted MR imaging in differential diagnosis between intracerebral necrotic tumors and cerebral abscesses. AJNR Am J Neuroradiol 20: 1252-1257.

3. Mathew JM, Rajshekhar V, Chandy MJ (1996) MRI features of neurosurgical gossypiboma: report of two cases. Neuroradiology 38: 468-469.

4. O'Connor AR, Coakley FV, Meng MV, Eberhardt SC (2003) Imaging of retained surgical sponges in the abdomen and pelvis. AJR Am J Roentgenol 180: 481-489.

5. Oto A, Remer EM, O'Malley CM, Tkach JA, Gill IS (1999) MR characteristics of oxidized cellulose (Surgicel). AJR Am J Roentgenol 172: 1481-1484.

6. Yuh-Feng T, Chin-Chu W, Cheng-Tau S, Min-Tsung T (2005) FDG PET CT features of an intraabdominal gossypiboma. Clin Nucl Med 30: 561-563. 